\title{
Real-time 3D-representation of time-resolved infrared thermographic data
}

\author{
by U. NETZELMANN, G. WALLE and G. DOBMANN
}

Fraunhofer-Institute for Non-Destructive Testing, University, Bldg. 37,

Saarbrücken, Fed. Rep. of Germany.

Abstract

A graphic workstation with volume-rendering software is used to visualize the cooling process of samples measured by time-resolved infrared thermography. The recorded data set, consisting of images of up to 80 frames with $320 \times 256$ pixels each, can be sliced in any desired plane in real time. Ray-tracing techniques are applied to achieve a comprehensive representation of inner structures of the specimen.

\section{Introduction}

When recording time-resolved infrared video signals, often large amounts of data are collected. Searching several megabytes of data for unknown samples can be a time-consuming procedure when loading frame by frame from files. Present systems are designed to display sequences of infrared images ( $x y$ planes) or to convert the measured data into a representation called thermal tomogram [1]. We think that it is desirable to visualize the evolution of the thermal signal on a line as a funtion of time, in order to see a spatial-temporal profile. This representation will show the depth dependence of inner structures of the sample in analogy to ultrasound B-scanning. In this contribution, we report on the application of modern graphic software for visualization of thermographic data.

\section{Experimental technique}

Our thermographic system (figure 1) is based on an infrared (IR) videocamera (Inframetrics 600 ) with a temperature resolution of 0.2 degrees and a frame rate of 25 per second. Telescope and microscope lenses are available. For excitation, a flash lamp with an energy of $6400 \mathrm{~J}$ and a pulse duration of $5 \mathrm{~ms}$ is used. The thermal response of the sample is recorded by an 8 bit real-time frame-grabber, which is able to store up to 80 frames in fixed or arbitrary spacing in time subsequent to the heating pulse. Each frame consists of $320 \times 256$ pixels. To show defects with higher contrast, it is possible to subtract the cooling curve of a reference sample from the cooling curve of a defective sample in the frame grabber.

Up to 6.5 megabytes of data are collected by the frame-grabber within a single cooling process and stored in the personal computer controlling the experiment. For the visualization, the frame data are written to a single file and transferred via an Ethernet link to the graphic workstation. 


\section{http://dx.doi.org/10.21611/qirt.1992.037}

\section{Visualization}

A SUN 4/370 workstation is used for the interactive visualization. The workstation is equipped with an application accelerator board with 8 megabytes of local memory, which is necessary to achieve the computing speed required for interactive operation. The software package (Voxvu, Sun Microsystems) is designed to work in real-time by mouse control.

The possibilities of the system are demonstrated for an artificial sample consisting of several voids in different depths in a plastic material (figure 2). The sample has four voids in depths of $0.15,0.30,0.45$ and $0.60 \mathrm{~mm}$, each of them has a size of $2 \mathrm{~mm} \times 2 \mathrm{~mm}$. 50 frames have been recorded and transferred to the workstation.

There are different techniques available to visualize the data measured. Generally, the data are represented as a block in perspective view, which is outlined in green in figure $A^{*}$. The top surface of the block corresponds to the IR-image obtained immediately after the flash pulse $(t=40 \mathrm{~ms}$ ), the total length of the time axis (which is the short edge of the block) is about $7 \mathrm{~s}$. The image frames have been sampled equally spaced on a $t^{2}$-scale, in order to achieve a linear relation between occurrence of structures and their depth under the surface.

In the slicing technique, the thermal signal is coded by a colour scale. The data block can be turned into any desired orientation. Looking into the data is possible by shifting one of the outer surfaces of the data block into the volume. One can scan through the volume in real-time with any desired plane and search the volume for inner structures. In figure $A$, an $x y$-plane at a time of about $1200 \mathrm{~ms}$ has been selected. The area imaged is about $12.5 \mathrm{~cm} \times 10 \mathrm{~cm}$. The test sample is appearing as the central rectangular feature in the plane. Three of the four voids are appearing at this time. When turning the cutting plane around by 90 degrees, one obtains a representation shown in figure $B$. Here, a spatial/temporal plane has been cut, which shows the depth distribution of the voids. The outer left and right columins in the cross section of figure $A$ are due to edge effects at the lateral faces of the sample, whereas the four central columns are showing the voids. Unfortunately, due to the limited dynamic range of acquisition system, there was the need to combine the full cooling sequence from several shots with adapted camera gain in different time domains, which causes the sudden color jump at about a quarter of the full time range displayed. In spite of these effects, one can observe the delayed appearance of the signal from the voids in different depths. As expected from the structure of the sample, there is an almost linear increase of the depth of the voids due to the $t^{2}$-representation. From the arrival times $t$ of the void signals and the known depths $d$, a thermal diffusivity $\alpha=8 \cdot 10^{-8} \mathrm{~m}^{2} / \mathrm{s}$ can be estimated from the relation $t=0.25 \mathrm{~d} / \alpha$.

Cutting the data block with arbitrarily inclined planes or with several planes simultaneously is possible. Positions inside the volume can be quantified with a video cursor.

An alternative representation can be achieved by using ray-casting techniques. Here, within a mathematical model rays are sent through the volume and are projected to the image plane. Depending on the imaging strategy, the maximum or the integrated value of the temperature signal is converted into a grey value, leading to an $\mathrm{X}$-ray like image. In a more sophisticated approach, different intervals of amplitude values are assigned to substances with selectable optical properties like color and transparency. This artificial object is illuminated by a moveable light source and a photorealistic image is produced. The ray-casting techniques allow one to produce comprehensive representations of an object with a complicated inner structure. In a further step, many perspective views with the object turned around slightly between each can be replayed as a movie of the rotating object. 


\section{Conclusion}

Application of modern data representation techniques allows one to extract useful information from large data sets. Advantages to earlier techniques are due to the real-time interactive operation and due to the higher image quality available. The software package used is universal, allowing to visualize data with any method generating volume data information. For example, we are using the same system for representation of ultrasound [2] and X-ray [3] volume data. In this work, only raw or subtracted IR data are used, but in the future visualization systems will become more useful for representation of reconstructed and inverted thermal data.

\section{REFERENCES}

[1] FAVRO (L. D.), AHMED (T.), CROWTHER (D.), JIN (H. J.), KUO (P. K.) and WANG (X.). Infrared thermal-wave studies of coatings and composites. Thermosense XIII, 290, 1991. [2] NETZELMANN (U.), HERZER (R.), STOLZ (H.) and ARNOLD (W.). - Volume acquisition and visualization of high-frequency ultrasound data. Proc. Acoustical Imaging 1991, Bochum, FRG, to be published by Plenum Press, New-York.

[3] MAISL (M.) and HERZER (R.). - Advanced 2D and 3D representation of high-frequency ultrasound and high resolution $X$-ray computed tomography data for inspection of modern materials. Proc. Int. Conf. on Monitoring and Predictive Maintenance of Plants and Structures 1992, May 17-20, 1992, Firenze, Italy, published by AIPnD, Brescia, 1992. 
http://dx.doi.org/10.21611/qirt.1992.037
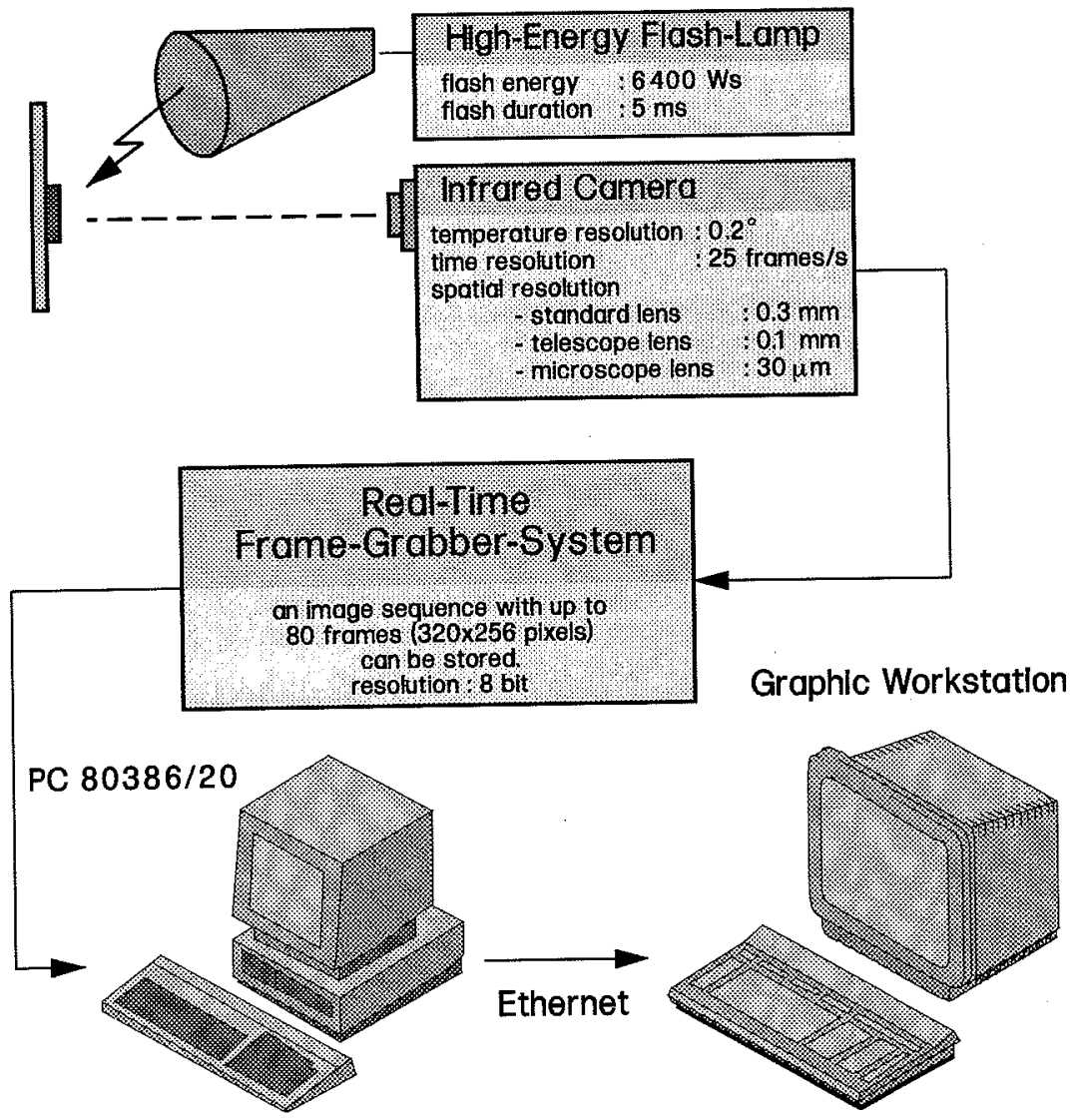

Fig. 1. - Block diagram of the experimental arrangement

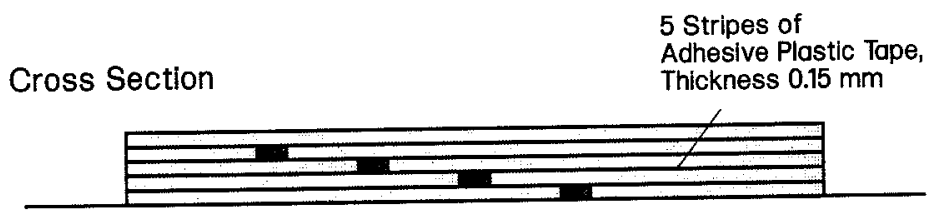

Metal Plate

Top View

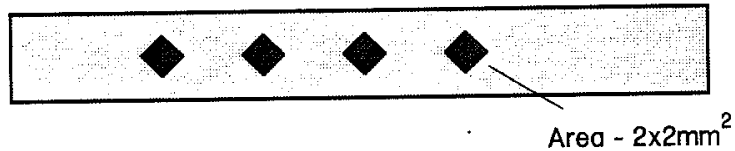

Fig. 2. - Sketch of the sample studied 